\title{
First autopsy analysis of a neovascularized arterial network induced by indirect bypass surgery for moyamoya disease: case report
}

\author{
Maki Mukawa, MD, ${ }^{1}$ Tadashi Nariai, MD, PhD, ${ }^{1}$ Motoki Inaji, MD, PhD, ${ }^{1}$ Natsumi Tamada, MD, ${ }^{1}$ \\ Taketoshi Maehara, MD, PhD, ${ }^{1}$ Yoshiharu Matsushima, MD, PhD, ${ }^{1}$ Kikuo Ohno, MD, PhD, ${ }^{1}$ \\ Mariko Negi, MD, PhD, ${ }^{2}$ and Daisuke Kobayashi, MD, PhD²
}

Departments of ${ }^{1}$ Neurosurgery and ${ }^{2}$ Human Pathology, Tokyo Medical and Dental University, Bunkyo-ku, Tokyo, Japan

\begin{abstract}
The object of this study was to analyze the pathology of collateral vessels newly induced by indirect bypass surgery for moyamoya disease (MMD). An autopsy analysis was conducted on a 39-year-old woman with MMD who had died of a brainstem infarction. The patient had undergone bilateral indirect bypass surgeries 22 years earlier. Sufficient revascularization via bilateral external carotid arterial systems was confirmed by cerebral angiography before her death. Macroscopic observation of the operative areas revealed countless meandering vessels on the internal surface of the dura mater connected with small vessels on the brain surface and in the subpial brain tissue. Notably, microscopic analysis of these vessels revealed the characteristic 3-layer structure of an arterial wall. This autopsy analysis was the first to confirm that indirect bypass surgery had induced the formation of a new arterial network (arteriogenesis) and that this network had been maintained for more than 20 years to compensate for the chronic cerebral ischemia caused by the MMD.
\end{abstract}

http://thejns.org/doi/abs/10.3171/2015.4.JNS15155

KEY WORDS moyamoya disease; encephaloduroarteriosynangiosis; EDAS; arteriogenesis; vascular disorders

$\Lambda$ TECHNIQUE for indirect bypass surgery was developed as a unique treatment strategy for juvenile patients with moyamoya disease (MMD). ${ }^{9,12}$ In this technique, a newly formed vascular network connects the external and internal carotid systems spontaneously without direct mechanical vessel-to-vessel sutures. Many recent studies have suggested that indirect bypass surgery is useful for MMD in adults as well as juveniles., ${ }^{1,2,21}$ The efficacy of the procedure for adult atherosclerotic intracranial occlusive disease has also been documented by an institute in the US. ${ }^{3}$ Our group previously reported that surgically induced vascularization is initiated by a chronic cerebral ischemic condition represented by an elevated oxygen extraction fraction (OEF), increased cerebral blood volume (CBV), or a highly prolonged mean transit time (MTT) ${ }^{8,16}$ A reliable quantitative measurement of cerebral circulation may help to predict whether neovascularization will occur.

We speculate that the mechanism of vascularization via indirect procedures may be adaptable to any type of chronic cerebral ischemia in general. Before now, however, there have been no pathological reports of vessels newly induced by indirect surgery. We have obtained the first pathological findings of such vessels and describe them in detail here.

\section{Case Report}

History and Examination

A 39-year-old Japanese woman had no familial history of MMD. At the age of 9 years, she suffered from infarctions of the left temporooccipital lobe. At the age of 17 she underwent operations at our institute for encephaloduroarteriosynangiosis (EDAS) in the bilateral middle cerebral artery territories and for encephalogaleosynangiosis (EGS) in the right posterior cerebral artery territory. She suffered no deficits or symptoms thereafter and later took on a fulltime job.

At the age of 39 she suddenly presented with left cerebellar ataxia. Diffusion-weighted MRI revealed infarc-

ABBREVIATIONS CBF = cerebral blood flow; $\mathrm{CBV}=$ cerebral blood volume; $\mathrm{CPP}=$ cerebral perfusion pressure; $\mathrm{MMD}=$ moyamoya disease; $\mathrm{MTT}=$ mean transit time; $\mathrm{OEF}=$ oxygen extraction fraction; STA = superficial temporal artery.

SUBMITTED January 16, 2015. ACCEPTED April 6, 2015.

INCLUDE WHEN CITING Published online September 25, 2015; DOI: 10.3171/2015.4.JNS15155. 
tions of the left cerebellum. One day after her admission, the infarctions spread throughout her midbrain. Magnetic resonance angiography and vertebral angiography revealed progressive occlusion of the terminal portion of the basilar artery. Revascularization via the bilateral indirect bypass supplies was well maintained (Fig. 1A and B). On the 48th hospital day, respiratory insufficiency and cardiac arrest suddenly developed, and the patient died within hours. An autopsy analysis of the cerebral vessels was performed 43 hours 9 minutes after her death.

\section{Pathological Findings}

Brain tissue was removed with the dura mater, bilateral temporal muscles, and right occipital galeal tissue (Fig. 1C). Temporal galeal tissues placed on the brain surface by indirect procedures had formed a uniform layer with the original dura mater and could not be independently distinguished. Bilateral superficial temporal arteries (STAs) ran through on the external surfaces of these tissue complexes (Fig. 1D). The internal surfaces of these tissues were bridged with the brain surface by an abundance of very fine cords that readily snapped apart during the dissection (Figs. 2A and 3A). Orange ink was injected into the STA running outside the dura mater and oozed out from the ends of the protruding cords on the internal surface of the dura mater.
In the microscopic examinations, arteries in the dura mater projected to the brain (Fig. 2B). A 3-layer structure was maintained in the walls of these arteries (Fig. 2C), but the media was thin, sparse, and mainly composed of smooth muscle actins interspersed with collagen fibers (Fig. 2D). Arteries in the subpial tissue were variable in diameter (Fig. 3B).

We previously described the pathologies underlying this patient's death in a report on the long-term clinical follow-up of juvenile patients with moyamoya treated by surgery. ${ }^{13}$

\section{Discussion}

This is the first autopsy report confirming the presence of a vascular network in MMD formed after an indirect bypass surgery performed many years earlier. This vascular network had preserved a stable cerebral blood supply for more than 20 years. Microscopic examination of the vascular network revealed that the characteristic 3-layer structure of arteries was maintained in the walls of numerous collateral vessels.

Several autopsy studies have been performed to analyze the pathology of the occluded internal carotid system and moyamoya vessels in patients with MMD.,6 To date, however, there have been no autopsy analyses of well-developed anastomotic vessels induced by indirect bypass
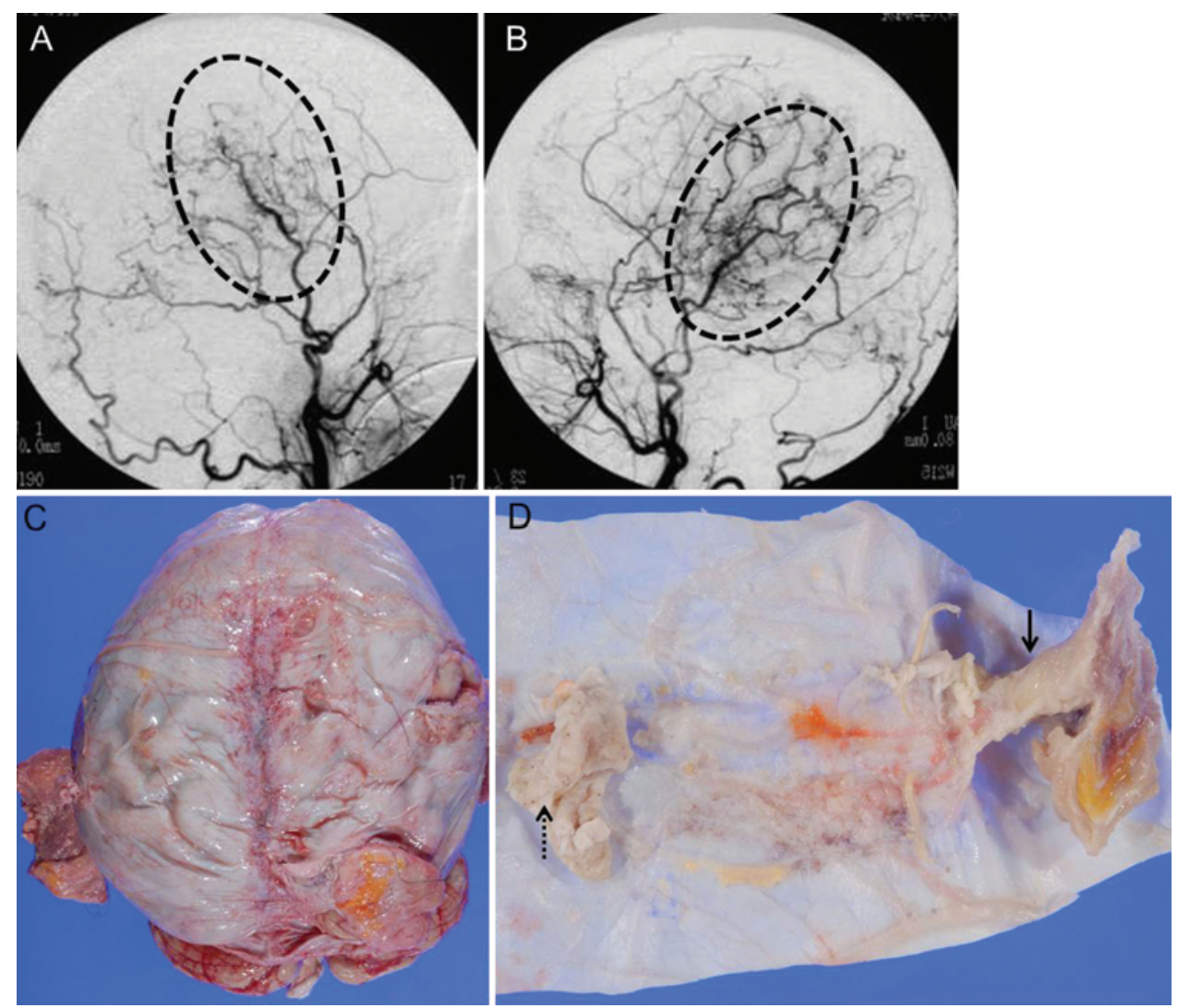

FIG. 1. Arterial phase on right (A) and left (B) external carotid artery angiographs obtained 16 days before the patient's death. The area of craniotomy - that is, the area examined in the present autopsy analysis-is roughly indicated by the ellipses. The brain specimen (C) is wrapped in dura mater. External side of dura mater (D). A superficial temporal artery flows in (solid arrow) and out (dotted arrow). Figure is available in color online only. 

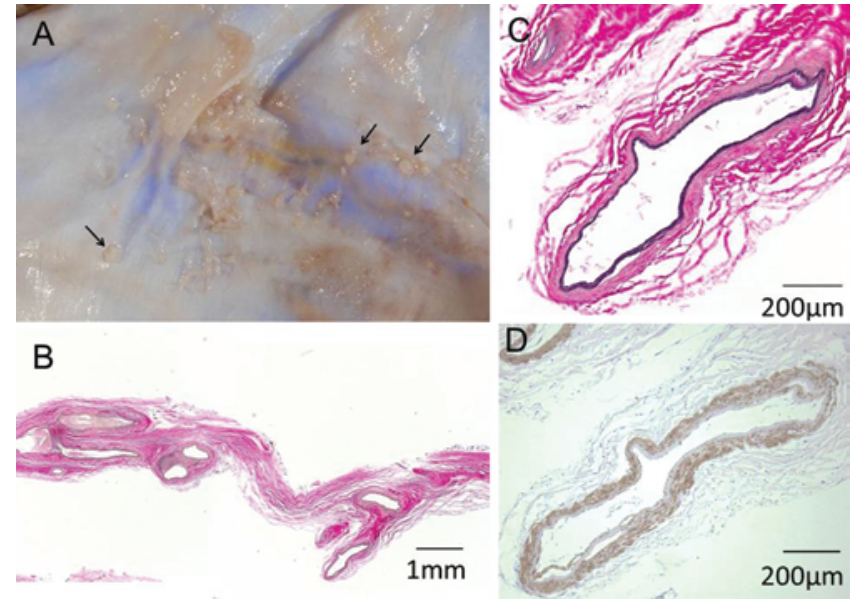

FIG. 2. Internal side of the dura mater (A), where the ends of many protruding cords can be seen (arrows). Numerous arteries run through the internal side of the dura mater (B). Some of these arteries sprout to the brain (C). The 3-layer structure is maintained in the vascular walls (D). The media is thin, sparse, and composed mainly of smooth muscle actins. It clearly differs in structure from the media of preexisting vessels formed naturally. Elastic van Gieson stain (B and $C$ ) and $\alpha$-smooth muscle actin stain (D).

surgery. Poor revascularization was reported in autopsy analyses of indirect bypasses in 2 patients who had undergone surgery for preexisting brain damage. ${ }^{10,20}$

Broadly speaking, 2 forms of vessel growth are known to compensate for tissue ischemia after birth: angiogenesis and arteriogenesis. ${ }^{5}$ Angiogenesis, a capillary sprouting triggered by hypoxia, results in higher capillary density. Arteriogenesis, a rapid proliferation of preexisting collateral arteries triggered by fluid shear stress, results in normal arteries. ${ }^{5}$ In cases of MMD, the mechanism of neovascularization after indirect bypass surgery has been considered as "angiogenesis,",, 16 although the histological etiology was unclear. Matsushima ${ }^{11}$ speculated that at the earliest stage of indirect anastomosis, spontaneous communication between the extracranial and intracranial arteries is formed by angiogenesis of wound healing in the granulation tissue of the operative wound and that the pressure gradient between the 2 arterial systems ensures the constant and early flow from the external carotid artery to the internal carotid artery systems. In a recent experimental study on revascularization after indirect anastomosis surgeries in pig models of chronic cerebral ischemia, Nakamura et al..$^{14}$ also concluded that functional revascularization required 2 steps: first, the development of tissue providing vascular beds (angiogenesis), and second, stimulus of fluid shear stress for artery-to-artery anastomosis (arteriogenesis).

In our microscopic analysis, vessels sprouting from the dura mater to the brain tissue clearly had the 3-layer structure characteristic of arteries, and they were not fragile capillaries. However, these arteries were apparently newly formed, as their media differed in structure from the media found in the other arteries formed naturally. The present result suggests that arteriogenesis played a definite role in the collateral network formation induced by the indirect bypass surgery. Our autopsy analysis may provide the first
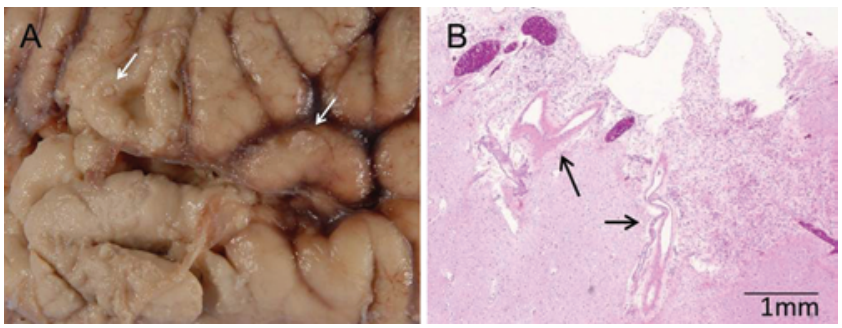

FIG. 3. Cords (arrows) on the brain surface (A). Arteries (arrows) of nonuniform diameter in the subpial brain tissue (B). H \& E stain (B). Figure is available in color online only.

clinical and histological evidence to support Matsushima's and Nakamura's theory.

Powers and colleagues ${ }^{17,18,19}$ summarized the stages of hemodynamic cerebral ischemia and the compensatory mechanisms against it. If cerebral perfusion pressure (CPP) falls, CBV increases to preserve the initial cerebral blood flow (CBF; Stage 1 in the Powers' classification). If CBV reaches maximum while CPP continues to progressively fall, the OEF rises (Stage 2). According to Powers' theory, a reduced CPP manifests as a prolonged MTT. Our former clinical study indicated that indirect bypass surgery induces the formation of good collateral vessels when the operative field has an extremely elevated CBV, abnormally high OEF, and extremely prolonged MTT. ${ }^{8,15,16}$ According to Powers' theory, all of these conditions coincide with a drastically reduced CPP. As stated earlier, arteriogenesis is initially triggered by fluid shear stress, a difference in perfusion pressure affecting a single tissue. We know that CPP influences the effects of indirect bypass surgery, as arteriogenesis takes part in the collateral network formation induced by the intervention. Once the indirect-bypass procedure has helped to form the connection between the brain surface and the external carotid artery system during wound healing (scar formation and angiogenesis), the arteriogenesis progresses from the external carotid artery system with normal CPP to the brain surface with extremely reduced CPP. This interpretation of the nature of indirect bypass surgery allows us to reasonably predict the degree of arteriogenesis inducible by surgical treatment based on our former studies.

We find this hypothesis attractive and believe that it explains many things without contradiction. We recognize, however, that the hypothesis represents only one way of thinking and is derived from only one autopsy study and one experimental study. Many of the mechanisms of vascularization from the initial stage of angiogenesis to the final stage of arteriogenesis will have to be confirmed by further studies combining experimental and clinical evidence. In any case, this topic may provide very useful information for understanding the compensatory mechanism of chronic cerebral ischemia of the human brain.

\section{References}

1. Bao XY, Duan L, Li DS, Yang WZ, Sun WJ, Zhang ZS, et al: Clinical features, surgical treatment and long-term outcome in adult patients with Moyamoya disease in China. Cerebrovasc Dis 34:305-313, 2012

2. Dusick JR, Gonzalez NR, Martin NA: Clinical and angio- 
graphic outcomes from indirect revascularization surgery for Moyamoya disease in adults and children: a review of 63 procedures. Neurosurgery 68:34-43, discussion 43, 2011

3. Dusick JR, Liebeskind DS, Saver JL, Martin NA, Gonzalez NR: Indirect revascularization for nonmoyamoya intracranial arterial stenoses: clinical and angiographic outcomes. J Neurosurg 117:94-102, 2012

4. Fukui M, Kono S, Sueishi K, Ikezaki K: Moyamoya disease. Neuropathology 20 (Suppl):S61-S64, 2000

5. Heil M, Eitenmüller I, Schmitz-Rixen T, Schaper W: Arteriogenesis versus angiogenesis: similarities and differences. J Cell Mol Med 10:45-55, 2006

6. Hosoda Y, Ikeda E, Hirose S: Histopathological studies on spontaneous occlusion of the circle of Willis (cerebrovascular moyamoya disease). Clin Neurol Neurosurg 99 (Suppl 2):S203-S208, 1997

7. Houkin K, Nakayama N, Kuroda S, Ishikawa T, Nonaka T: How does angiogenesis develop in pediatric moyamoya disease after surgery? A prospective study with MR angiography. Childs Nerv Syst 20:734-741, 2004

8. Ishii Y, Nariai T, Tanaka Y, Mukawa M, Inaji M, Maehara T, et al: Practical clinical use of dynamic susceptibility contrast magnetic resonance imaging for the surgical treatment of moyamoya disease. Neurosurgery 74:302-309, 2014

9. Karasawa J, Kikuchi H, Furuse S, Sakaki T, Yoshida Y: A surgical treatment of "moyamoya" disease "encephalo-myo synangiosis". Neurol Med Chir (Tokyo) 17:29-37, 1977

10. Kono S, Oka K, Sueishi K, Sonobe M: Histopathological studies on spontaneous vault moyamoya and revascularized collaterals formed by encephalomyosynangiosis. Clin Neurol Neurosurg 99 (Suppl 2):S209-S212, 1997

11. Matsushima Y: Moyamoya disease, in Youmans JR (ed): Youmans Neurological Surgery, ed 4. Philadelphia: Saunders, 1996

12. Matsushima Y, Fukai N, Tanaka K, Tsuruoka S, Inaba Y, Aoyagi M, et al: A new surgical treatment of moyamoya disease in children: a preliminary report. Surg Neurol 15:313320, 1981

13. Mukawa M, Nariai T, Matsushima Y, Tanaka Y, Inaji M, Maehara T, et al: Long-term follow-up of surgically treated juvenile patients with Moyamoya disease. J Neurosurg Pediatr 10:451-456, 2012

14. Nakamura M, Imai H, Konno K, Kubota C, Seki K, Puentes $\mathrm{S}$, et al: Experimental investigation of encephalomyosynangiosis using gyrencephalic brain of the miniature pig: histopathological evaluation of dynamic reconstruction of vessels for functional anastomosis. Laboratory investigation. J Neurosurg Pediatr 3:488-495, 2009
15. Nariai T, Matsushima Y, Imae S, Tanaka Y, Ishii K, Senda M, et al: Severe haemodynamic stress in selected subtypes of patients with moyamoya disease: a positron emission tomography study. J Neurol Neurosurg Psychiatry 76:663-669, 2005

16. Nariai T, Suzuki R, Matsushima Y, Ichimura K, Hirakawa K, Ishii K, et al: Surgically induced angiogenesis to compensate for hemodynamic cerebral ischemia. Stroke 25:1014-1021, 1994

17. Powers WJ: Cerebral hemodynamics in ischemic cerebrovascular disease. Ann Neurol 29:231-240, 1991

18. Powers WJ, Grubb RL Jr, Raichle ME: Physiological responses to focal cerebral ischemia in humans. Ann Neurol 16:546-552, 1984

19. Powers WJ, Press GA, Grubb RL Jr, Gado M, Raichle ME: The effect of hemodynamically significant carotid artery disease on the hemodynamic status of the cerebral circulation. Ann Intern Med 106:27-34, 1987

20. Sayama I, Fukasawa H, Yasui N, Suzuki A: [Child with moyamoya disease after bypass surgery. Report of an autopsy case.] Neurol Med Chir (Tokyo) 25:975-980, 1985 (Jpn)

21. Starke RM, Komotar RJ, Hickman ZL, Paz YE, Pugliese AG, Otten ML, et al: Clinical features, surgical treatment, and long-term outcome in adult patients with moyamoya disease. Clinical article. J Neurosurg 111:936-942, 2009

\section{Disclosure}

The authors report no conflict of interest concerning the materials or methods in this study or the findings specified in this paper.

\section{Author Contribution}

Conception and design: Nariai. Acquisition of data: Nariai, Mukawa, Ito, Matsushima, Ohno, Negi, Kobayashi. Analysis and interpretation of data: Nariai, Mukawa, Inaji, Matsushima, Ohno, Negi, Kobayashi. Drafting the article: Mukawa, Kobayashi. Critically revising the article: Nariai. Reviewed submitted version of manuscript: all authors. Approved the final version of the manuscript on behalf of all authors: Nariai. Administrative/technical/material support: Matsushima, Ohno.

\section{Correspondence}

Tadashi Nariai, Department of Neurosurgery, Tokyo Medical and Dental University, 1-5-45 Yushima, Bunkyo-ku, Tokyo 113, Japan. email: nariai.nsrg@tmd.ac.jp. 